\title{
Modelando Requisitos de RH para Análise do Trabalho em Grupo
}

\author{
Juliana Cesar Sirena Machado ${ }^{1}$, Henrique Prado de Sá Sousa ${ }^{2}$ \\ ${ }^{1}$ Departamento de Computação - Universidade Federal Rural do Rio de Janeiro \\ (UFRRJ) \\ Rodovia BR 465, Km 07, s/n - Zona Rural, Seropédica - RJ, 23890-000 \\ ${ }^{2}$ Departamento de Informática - Universidade Federal do Estado do Rio de Janeiro \\ (UNIRIO) \\ Av. Pasteur, 296 - Urca - Cep 22290-240 \\ juliana.sirena@gmail.com, hsousa@uniriotec.br
}

Abstract. In organizations, goals can be achieved by one or more actors, who contribute in different ways. Analyzing the contribution of these individuals is important to maintaining the organization's alignment. In this work, a research was carried out for specific competences for acting in group work and variables that are specific in the interaction of group work were identified. Subsequently, the process of automating the use of these variables was carried out, creating an extension of the GPI-HR language, specific for group work modeling. Models are then presented to exemplify the use of these variables in the alignment of human resources groups and to demonstrate the new features developed, which demonstrate that the consideration of these new elements can allow analysis of human resources alignment for group activities.

Resumo. Nas organizações, objetivos podem ser realizados por um ou mais atores, que contribuem de formas diferentes. A análise da contribuição desses indivíduos é importante para manter o alinhamento da organização. Neste trabalho, foi realizada a pesquisa por competências especificas para a atuação em trabalhos em grupo e foram identificadas variáveis que são especificas na interação do trabalho em grupo. Posteriormente foi realizado o processo de automatização do uso dessas variáveis, criando uma extensão da linguagem GPI-HR, específica para modelagem de trabalho em grupo. Posteriormente são apresentados modelos para exemplificar o uso dessas variáveis no alinhamento de grupos de recursos humanos e para demonstrar as novas funcionalidades desenvolvidas, os quais demonstram que a consideração destes novos elementos pode permitir análise do alinhamento de recursos humanos para atividades em grupo.

\section{Introdução}

A análise do alinhamento de Recursos Humanos (RH), é uma importante ferramenta de tomada de decisão das organizações, permitindo a análise da contribuição dos colaboradores e adequação de processos de trabalho, para alcançar o objetivo estratégico da organização [Del Corso et al., 2014]. 
A análise do alinhamento dos recursos humanos de uma organização demanda ferramentas específicas que evidenciem não só as competências individuais, mas também as competências inerentes à implementação de tarefas colaborativas, e como essas relações contribuem com o sucesso dos objetivos estabelecidos.

Para tarefas que demandam a colaboração entre indivíduos, a complexidade do alinhamento pode aumentar. São necessárias competências sociais específicas que habilitam o desempenho satisfatório de um grupo de trabalho. A ausência de requisitos para o trabalho em grupo, ou presença de características conflitantes podem comprometer a realização da tarefa.

A modelagem organizacional contribui com o registro de informações relevantes que auxiliam a compreender as complexas interações entre os componentes da organização, facilitando a sua análise e auxiliando em decisões [Pádua et al., 2004].

Algumas linguagens de modelagem organizacional apresentam recursos para modelar conceitos pertinentes aos recursos humanos (ex. [Singh e Woo, 2009] e [Sousa e Leite, 2017], mas não exploram variáveis específicas do processo de trabalho em grupo. A introdução desses conceitos à uma linguagem de modelagem pode auxiliar o registro e análise de informações presentes na interação de competências individuais quando avaliados em trabalho em grupo.

O objetivo deste trabalho foi identificar variáveis relevantes para a análise do trabalho em grupo e, posteriormente, integrá-las a uma linguagem de modelagem organizacional. Utilizamos a linguagem GPI-HR (Goal, Process, Indicators - Human Resources), uma extensão da linguagem GPI, desenvolvida para auxiliar na análise do alinhamento de RH. A linguagem permite o mapeamento de competências de indivíduos (RHs) e competências requeridas aos atores organizacionais [Sousa, 2017].

A linguagem GPI-RH foi utilizada como base para a inclusão de novos elementos que apoiem a análise do alinhamento de recursos humanos em processos de trabalho em grupo. Esta linguagem foi escolhida por trazer conceitos relevantes a análise de RH, por exemplo, o conceito de competências. A extensão da linguagem GPI-HR também inclui a evolução do mecanismo de análise de RH para melhor representar as relações resultantes do trabalho em grupo.

Para o desenvolvimento dessa extensão e sua implementação, foi elicitado um conjunto de competências de RH relevantes para o contexto de grupos de trabalho e requisitos específicos de trabalho, como grau de importância das competências e escalas de trabalho dos colaboradores envolvidos em um projeto. Foi proposto um método de propagação automática de marcadores, utilizando pesos para representar com maior fidelidade as diferentes contribuições individuais nos trabalhos colaborativos. Este método foi implementado na ferramenta GPI-HR ${ }^{1}$.

\footnotetext{
${ }^{1}$ Disponível em gpi.uniriotec.br.
} 


\section{Definindo requisitos de RH para o trabalho em grupo}

$\mathrm{O}$ primeiro passo para identificar os requisitos de $\mathrm{RH}$ para o trabalho em grupo foi a identificação das características individuais relevantes para o trabalho em grupo. Esse conjunto de características não é uma lista definitiva, mas uma seleção composta por elementos que são bastante referenciados por autores como requisitos para o bom desempenho de indivíduos em trabalhos em grupo. Esta lista foi utilizada neste trabalho, mas pode ser alterada, sem impactos à proposta.

Descrevemos estas características a seguir: A Comunicabilidade consiste na capacidade dos colaboradores de interagir de maneira positiva e eficaz [Stevens e Campion, 1994]. A Solução de Problemas é a capacidade de conduzir o esforço conjunto para manter o foco na solução de problemas [Stevens e Campion, 1994]. A Flexibilidade representa capacidade de adaptação à cada situação, a fim de retornos específicos [Chatenier, 2010]. O Respeito é a capacidade de adotar um comportamento adequado à organização, além da valorização da diversidade de opiniões entre as pessoas [Jehn e Bezrukova, 2004]. O Comprometimento é capacidade de maximizar os resultados obtidos pela organização, além de assegurar os esforços com o grupo em quaisquer situações [Chatenier, 2010]. A Clareza é a capacidade de análise e síntese de informações e explicitação de ideias aos membros do grupo [Goyal e Akhilesh, 2007]. A Ética é o respeito à diversidade e o atendimento de normas e diretrizes regulamentares [Thomas $\mathrm{e}$ Pender, 2008, p., tradução nossa]. A Responsabilidade é a forma com que o colaborador se posiciona fielmente para a realização de atividades. [Odelius et al., 2016]. A Colaboração/Cooperatividade representa a capacidade de estabelecer redes de transmissão de conhecimentos, além do aperfeiçoamento de ideias a partir da contribuição de outros colaboradores [Chatenier, 2010].

Duas outras variáveis importantes para caracterizar a dinâmica da interação de grupos de trabalho foram identificadas. A primeira é referente ao trabalho síncrono ou assíncrono, o que interfere na configuração da equipe e nos requisitos de trabalho (essa variável não é explorada no exemplo apresentado neste artigo por questões de espaço). A segunda variável é o grau de relevância de indivíduos e/ou de competências específicas as quais podem possuir a possibilidade de interferir no resultado do trabalho [Peduzzi, 2001].

\section{Evolução da linguagem GPI-HR}

\subsection{A linguagem GPI-HR}

O GPI-HR integra conceitos específicos do domínio de RH para permitir a avaliação do potencial da estrutura de RH em satisfazer aos requisitos da organização. Para isso, são modelados os requisitos de RHs, os quais são expressos como competências. Por sua vez, as competências são descritas pelo Perfil de Competências composto por um conjunto de Conhecimentos, Habilidades e Aptidões (CHA) [Stevens e Campion, 1994]. Esse conjunto de atributos representam as dimensões cognitiva, psicomotora e afetiva do indivíduo, e podem ser aplicadas durante a realização de atividades. [Fleury e Fleury, 2001] 
Para que um indivíduo seja capaz de atingir uma competência, ele deve possuir os requisitos $\mathrm{CHA}$ e alcançar determinado grau de qualidade esperado na prática das ações que implementam a competência. A avaliação do alinhamento de RH se baseia na modelagem de competências necessárias para que determinado ator organizacional possa lidar com suas responsabilidades. O mapa destas competências forma um perfil para o determinado papel organizacional, nomeado como Perfil Requerido.

O Perfil Requerido, quando instanciado para um indivíduo, gera as medições que avaliam o seu alinhamento aos requisitos organizacionais, e é chamado de Perfil Real. A partir do Perfil Real é possível observar a presença de desalinhamento. Os marcadores denotam o resultado das medições que são propagados aos demais elementos dos modelos através de seus relacionamentos. Neste trabalho, restringiremos os exemplos apenas aos modelos de Perfil Requerido, nos quais estão presentes os elementos aqui propostos.

\subsection{Modelagem de perfis de competências de grupo}

Os perfis de competência são representados como um elemento qualitativo, que pode ser medido de acordo com os quesitos de um avaliador, e seu grau é variável, se beneficiando das contribuições que recebe. A representação dos conceitos de CHA é feita por elementos gráficos específicos e representam operacionalizações aos respectivos Perfis. Podem ser relacionados através de uma relação "E", denotando ser um elemento obrigatório; ou através de uma relação de contribuição positiva, denotando ser um elemento opcional, mas que auxilia à competência a qual descreve. A Tabela 1 apresenta a notação para a modelagem do Perfil Requerido e Perfil Real.

Tabela 1 - Notação para modelagem do Perfil Requerido/Real

\begin{tabular}{|c|c|}
\hline Notação & Definição \\
\hline Perfil & $\begin{array}{l}\text { Representa um Perfil que é instanciado para detalhar determinada } \\
\text { competência em termos de seus requisitos de CHAs. }\end{array}$ \\
\hline Conhecimento $\mathrm{C}$ & Representa um conhecimento relacionado à um Perfil de Competência. \\
\hline $\begin{array}{ll}\text { Habilidade } & \oplus \\
\end{array}$ & Representa uma habilidade relacionada à um Perfil de Competência. \\
\hline \begin{tabular}{ll|} 
Aptidão & \\
\end{tabular} & Representa aptidão relacionada à um Perfil de Competência. \\
\hline Competência & $\begin{array}{l}\text { Representa uma competência. É opcional a sua representação no } \\
\text { modelo de Perfil Real/Requerido. }\end{array}$ \\
\hline & Representa uma contribuição positiva. Denota um “OU”. \\
\hline+ & Representa uma decomposição. Denota um “E”. \\
\hline & $\begin{array}{l}\text { Relacionamento que denota "detalhamento de requisitos" entre um Perfil } \\
\text { de Competência e sua respectiva Competência. }\end{array}$ \\
\hline
\end{tabular}

Para diferenciar os CHAs de trabalho em grupo em perfis que apresentem outros CHAs técnicos, foi adicionado um "G" do lado esquerdo dos elementos de CHAs (Figura 1). Essa marcação é importante para permitir a análise de alinhamento de forma separada. 


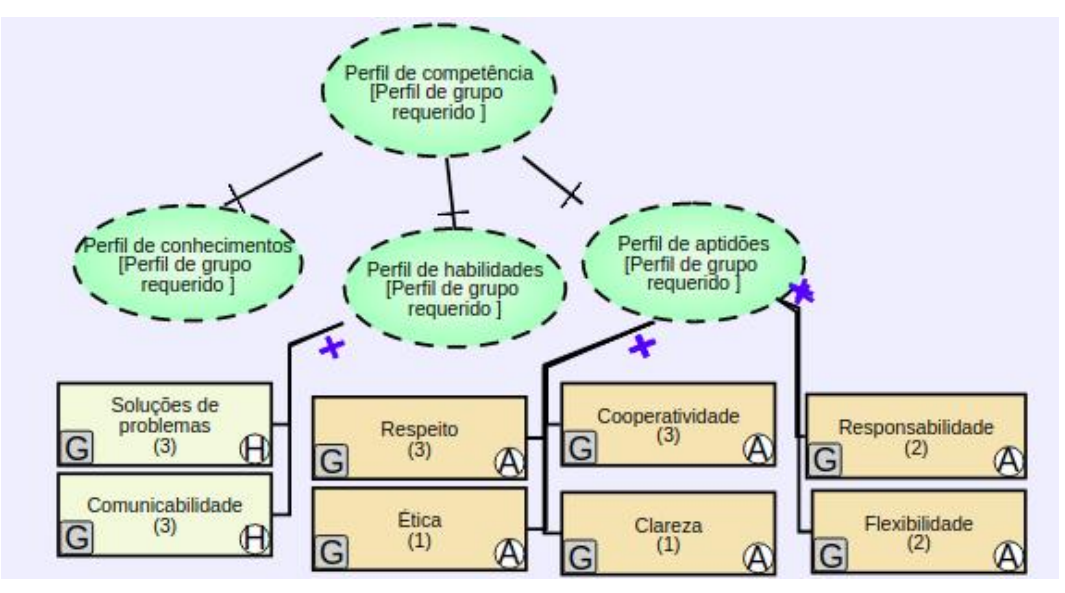

Figura 1 - Perfil requerido para trabalho em grupo

\subsection{Propagação ponderada de indicadores em grupos de trabalho}

A marcação de indicadores da linguagem GPI foi aprimorada, permitindo no mesmo modelo CHA a propagação de indicadores individuais e de grupo. Essa verificação é realizada através de dois métodos principais de atualização dos indicadores, que realizam a comparação entre os elementos do modelo CHA, a fim de estabelecer os níveis de hierarquia entre os elementos do modelo e permitir a propagação de marcadores pelo método da média ponderada ou pelo método do pior caso.

Esse método verifica o valor da média ponderada dos elementos CHA, e em seguida propaga para o elemento superior o valor resultante da média, conforme valores apresentados na tabela 2 .

Tabela 2. Valores utilizados para calcular a média ponderada dos indicadores do modelo GPI-HR

\begin{tabular}{|l|c|c|}
\hline Condição para atualizar marcador & $\begin{array}{c}\text { Valor para } \\
\text { cálculo }\end{array}$ & Cor \\
\hline Média ponderada maior do que o valor "0,6" & 1 & Azul \\
\hline $\begin{array}{l}\text { Média ponderada maior ou igual ao valor "0,1" e menor do que o valor } \\
\text { "0,6" }\end{array}$ & 0,5 & Verde \\
\hline $\begin{array}{l}\text { Média ponderada maior ou igual ao valor "-0,6" e menor do que o valor } \\
\text { "0,1" }\end{array}$ & $-0,5$ & Amarelo \\
\hline Média ponderada menor do que o valor "-0,6" & -1 & Vermelho \\
\hline
\end{tabular}

\section{Exemplo de análise de alinhamento de grupo de RH}

Foi elaborado um exemplo de modelagem para o trabalho em grupo, definidos a partir das descrições realizadas pelo Supervisor da área, visando complementar a análise do alinhamento de recursos humanos que foram mapeados para os cargos de Programador, Engenheiro de Requisitos e Administrador de Banco de Dados, são, respectivamente, Eng. De Requisitos Maria, DBA Fernanda e Programador Carlos (Figura 2, Figura 3 e Figura 4). 


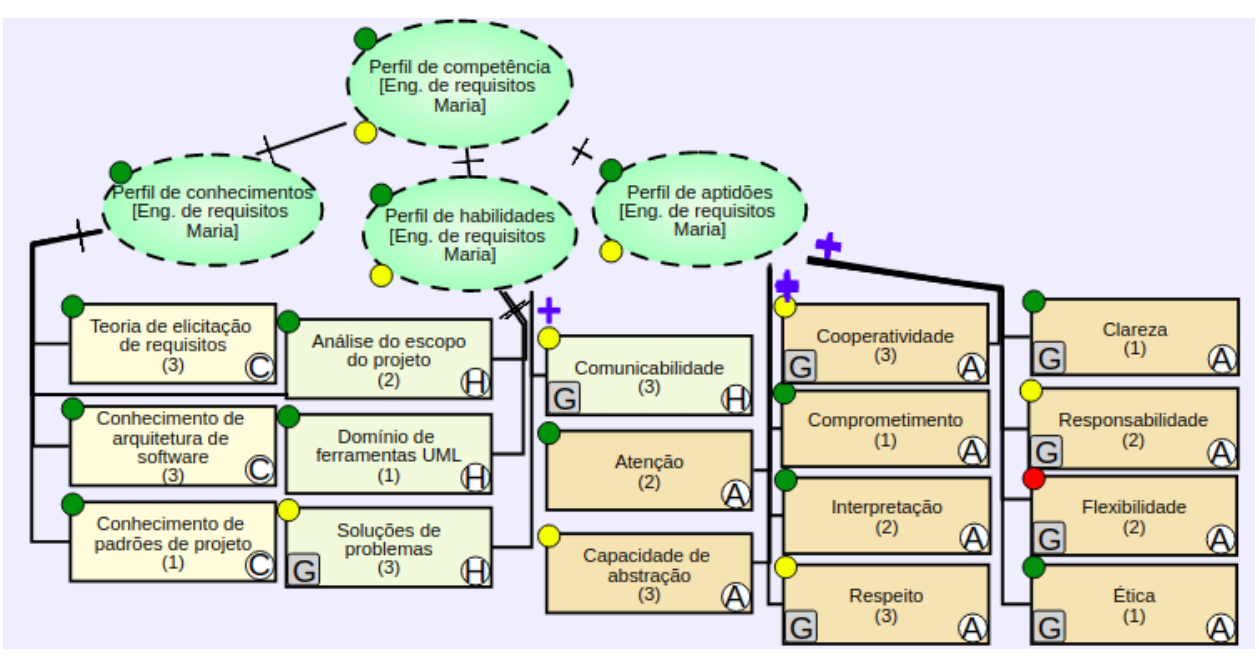

Figura 2 - Perfil Real da colaboradora Maria

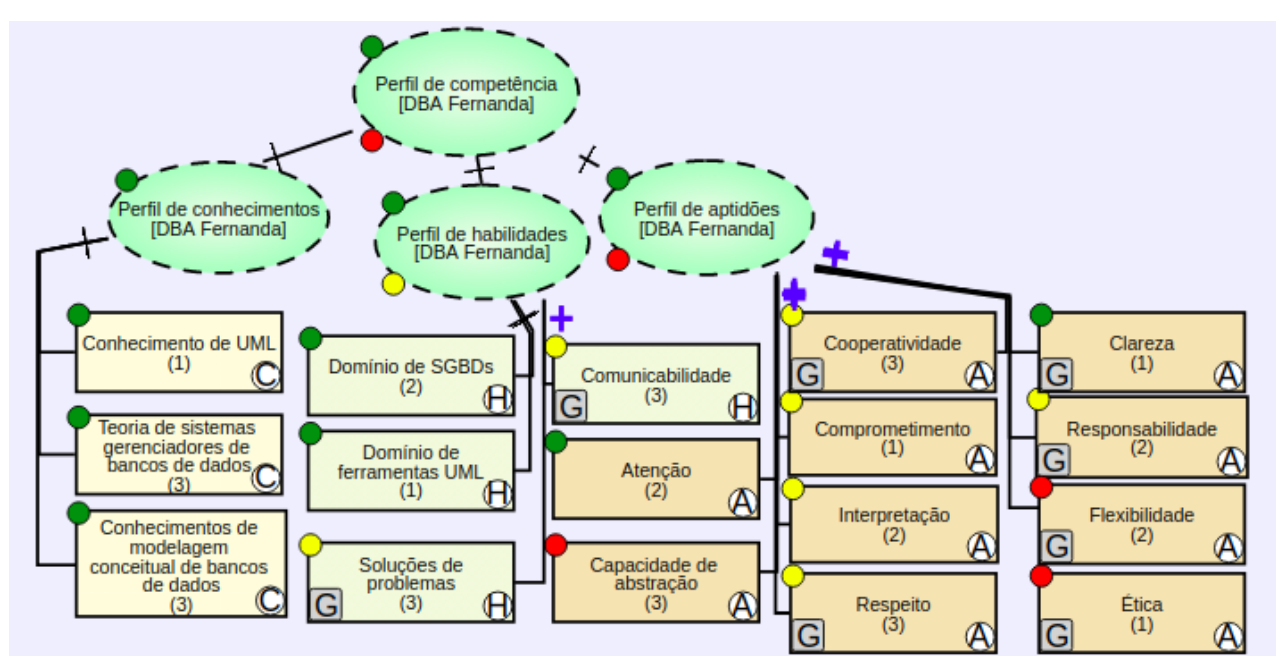

Figura 3 - Perfil Real da colaboradora Fernanda

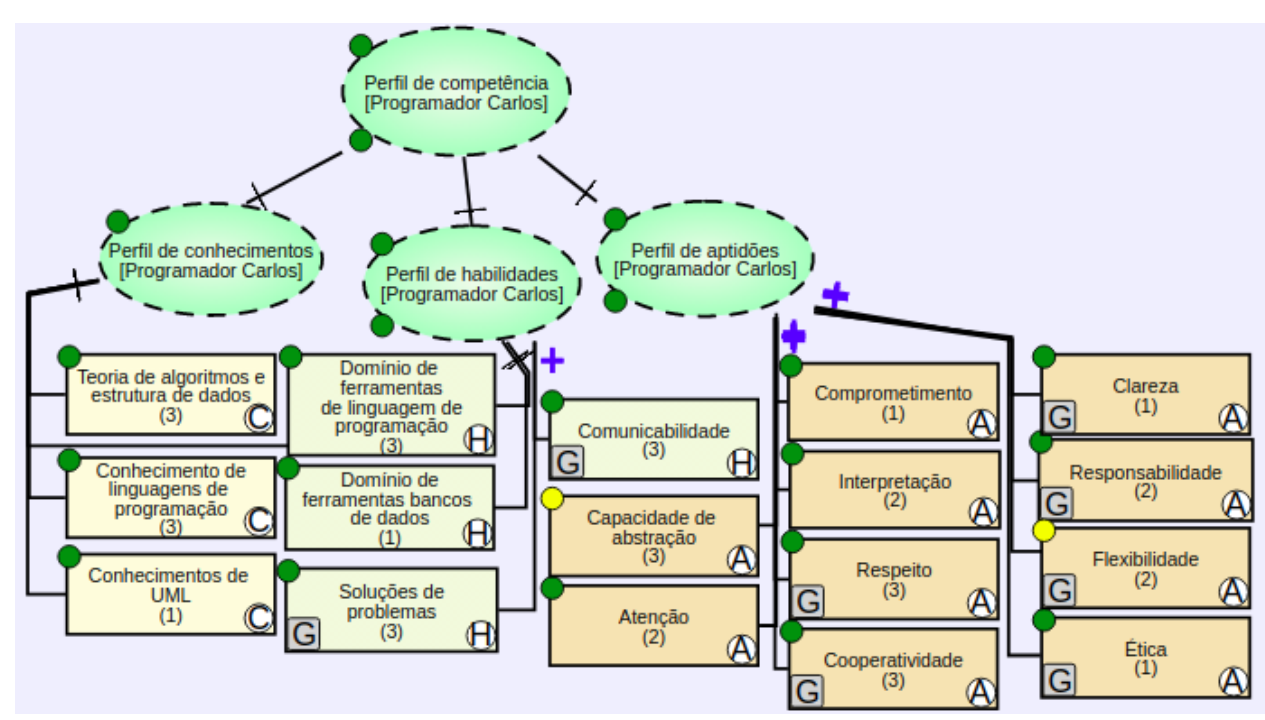

Figura 4 - Perfil Real do colaborador Carlos 
Para a realização da tarefa de desenvolvimento de uma nova funcionalidade de software, é necessário que os colaboradores possuam principalmente o alinhamento de competências específicas individuais, como conhecimento de "conhecimento de linguagem de programação", para o colaborador Carlos e de "Teoria de sistemas de bancos de sistemas gerenciadores de bancos de dados", para a colaboradora Fernanda. Por isso, essas competências estão com peso três tornando a sua contribuição de maior impacto para a propagação dos marcadores.

Ao analisar as competências de trabalho em grupo, a Engenheira de Requisitos Maria apresenta desalinhamento parcial de competência essenciais para a interação na realização da tarefa, como "respeito", "cooperatividade" e "responsabilidade", além do desalinhamento total da competência "flexibilidade", já o programador Carlos apresenta o alinhamento da maioria dessas competências, com exceção de "flexibilidade". Ao calcularmos os impactos desses colaboradores para as tarefas, foram propagados marcadores de desalinhamento parcial (cor amarela) para as tarefas em equipe e alinhamento para as tarefas individuais (cor verde) da colaboradora Maria.

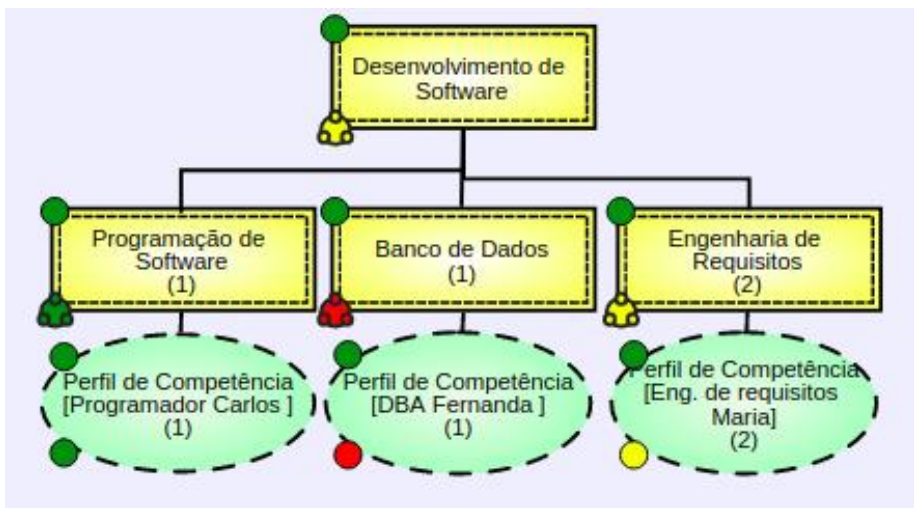

Figura 5 - Interação entre perfis de colaboradores

A figura 5 permite analisar a contribuição da interação entre os perfis dos três colaboradores envolvidos na realização do objetivo "Desenvolvimento de software". É possível, através da ponderação pelo método da média ponderada atenuar ou reduzir os desalinhamentos do modelo de competências. Para o modelo de exemplo desse trabalho, o resultado final da propagação de marcadores, evidenciou um desalinhamento parcial, devido à alguns resultados provenientes de dois colaboradores.

Os resultados encontrados nesse trabalho podem ser aplicados aos processos de tomada de decisão, pelo setor de RH da organização. Os desalinhamentos encontrados podem ser utilizados como insumo para melhorias de processo, tornando possível a busca pelo alinhamento organizacional.

\section{Conclusão}

Este trabalho propôs desenvolver mecanismos para auxiliar a modelagem e análise do alinhamento de recursos humanos atuando em tarefas de grupo, através da identificação de elementos da literatura de RH, que permitem analisar o relacionamento entre indivíduos. 
Algumas competências relevantes para a literatura de $\mathrm{RH}$ foram identificadas formando um conjunto inicial para auxiliar a análise dos perfis de competência para trabalho em grupo. O método de propagação por média ponderada permitiu diferenciar as contribuições de cada colaborador de acordo com o grau de relevância de determinadas competências.

O resultado da propagação de competências individuais e de grupo são propagados separadamente. Em trabalhos futuros, pretendemos desenvolver mecanismos de integração entre esses resultados para simplificar a análise do alinhamento de RH.

\section{Referências}

Chateier, E. du, et. al. (2010). "F. Identification of competencies for professionals in open innovation teams". R\&D Management, 271-280.

Del Corso, J. M, et al. (2014). "Gestão estratégica de recursos humanos: identificando o processo de alinhamento estratégico". Tourism \& Management Studies, v. 10, n. ESPECIAL, p. 49-57.

Fleury L. T. M. e Fleury A. (2001). "Construindo o Conceito de Competência”. RAC, Edição Especial, Curitiba.

Goyal, A., e Akhilesh, K. B. (2007). "Interplay among innovativeness, cognitive intelligence, emotional intelligence and social capital of work teams". Team Performance Management: An International Journal, 206-226.

Jehn, K. A. e Bezrukova, K. (2004). "A field study of group diversity, workgroup context, and performance". Journal of Organizational Behavior, 703-729.

Odelius, C.C., et al. (2016). "Atitudes e Habilidades Sociais para Trabalho em Equipe: Desenvolvimento de uma Escala”. RAC, Rio de Janeiro, v. 20, n. 2,p. 181 - p. 186.

Pádua, S I. D. de., et al. (2004). "Modelagem organizacional: captura dos requisitos organizacionais no desenvolvimento de sistemas de informação". Gestão \& Produção, v. 11, n. 2, p. 197-209.

Peduzzi, M. (2001) "Equipe multiprofissional de saúde: conceito e tipologia”. Revista de saúde pública, v. 35, n. 1, p. 103-109.

Singh, S. N. e Woo, C. (2009). "Investigating business-IT alignment through multidisciplinary goal concepts", Requirements Engineering 14.3, 177-207.

Sousa, H. P. S. e Leite, J. C. S. P. (2017). “Toward an organizational alignment modeling language: the Human Resource competency perspective".

Stevens, M. J. e Campion, M. A. (1994). "The Knowledge, Skill, and Ability Requirements for Teamwork: Implications for Human Resource Management". Journal of Management, 503-530.

Thomas, R. V., e Pender, D. A. (2008). "Association for Specialists in Group Work: Best Practice Guidelines 2007 Revisions”. The Journal for Specialists in Group Work, 33(2), 111-117. 